\title{
TOLERÂNCIA DE GENÓTIPOS DE SOJA AO ALUMÍNIO EM SOLUÇÃO'1
}

\author{
ORIVAL GASTÃO MENOSSO ${ }^{2}$, JOSÉ ANTONIO COSTA ${ }^{3}$, IBANOR ANGHINONI ${ }^{3}$ e HUMBERTO BOHNEN ${ }^{3}$
}

\begin{abstract}
RESUMO - O conhecimento da reação dos genótipos de soja (Glycine max L.) ao alumínio (Al), existente em solos ácidos, é indispensável para sua utilização em programas de melhoramento. Foram desenvolvidos, inicialmente, ensaios com o objetivo de identificar os níveis de $\mathrm{Al}$ e cálcio $(\mathrm{Ca}) \mathrm{em}$ soluções diluídas, e da melhor época para a medição dos parâmetros do sistema radicular para a separação dos genótipos de soja em relação à tolerância ao Al. Numa etapa seguinte, os ensaios objetivaram avaliar a tolerância ao Al de cultivares de soja brasileiras, de linhagens de interesse para o melhoramento, e de cultivares-padrões norte-americanas ao Al. Foi utilizado o nível de $0,2 \mathrm{mg} \mathrm{L}^{-1} \mathrm{de} \mathrm{Al} \mathrm{em}$ solução com $50 \mathrm{mg} \mathrm{L}^{-1}$ de Ca e determinado o incremento no comprimento da raiz primária até o nono dia. Dos 148 genótipos de soja testados, 21 foram tolerantes ao Al: Biloxi, Bragg, BRAS85-1736, BRAS86-3672, BR-13 (Maravilha), BR-37, Cobb, EMGOPA-302, EMGOPA-304 (Campeira), FT-1, FT-5 (Formosa), FT-6 (Veneza), FT-Guaíra, FT-Manacá, IAS 4, IPAGRO-21, Ivaí, MSBR-17 (São Gabriel), OCEPAR 6, Planalto e Tiaraju. Os genótipos agrupados como de tolerância intermediária foram em número de 73, e os sensíveis foram em número de 54.
\end{abstract}

Termos para indexação: Glycine max, cálcio, absorção, raízes.

\section{TOLERANCE OF SOYBEAN GENOTYPES TO ALUMINUM IN SOLUTION}

\begin{abstract}
The knowledge of the soybean (Glycine max L.) genotypes reaction to aluminum (Al) present in acid soils is important for the development of efficient breeding programs. A series of experiments were carried out to determine the levels of $\mathrm{Al}$ and calcium $(\mathrm{Ca})$ in low salt solutions and the most appropriate time for screening. The next experiments were conducted to rank Brazilian soybean cultivars and breeding lines according to their tolerance to $\mathrm{Al}$ with some American cultivars, considered as standards. A solution with $0.2 \mathrm{mg} \mathrm{L}^{-1}$ of $\mathrm{Al}$ and $50 \mathrm{mg} \mathrm{L}^{-1}$ of Ca was used and the primary root length obtained in the ninth day was utilized to screen 148 soybean genotypes for Al tolerance. Among these genotypes, 21 were Al-tolerant : Biloxi, Bragg, BRAS85-1736, BRAS86-3672, BR-13 (Maravilha), BR-37, Cobb, EMGOPA-302, EMGOPA-304 (Campeira), FT-1, FT-5 (Formosa), FT-6 (Veneza), FT-Guaíra, FT-Manacá, IAS 4, IPAGRO-21, Ivaí, MSBR-17 (São Gabriel), OCEPAR-6, Planalto and Tiaraju. Seventy three genotypes had intermediate tolerance and 54 were non-tolerant to $\mathrm{Al}$.
\end{abstract}

Index terms: Glycine max, calcium, absorption, roots.

\section{INTRODUÇÃO}

O desempenho da cultura da soja [Glycine $\max ($ L.) Merr.] em solos com problemas nutricionais

\footnotetext{
${ }^{1}$ Aceito para publicação em 12 de janeiro de 2000.

Extraído da tese de doutorado apresentada pelo primeiro autor à Faculdade de Agronomia, Universidade Federal do Rio Grande do Sul (UFRGS), Porto Alegre, RS.

${ }^{2}$ Eng. Agrôn., Dr., Embrapa-Centro Nacional de Pesquisa de Soja (CNPSo), Caixa Postal, 231, CEP 86001-970 Londrina, PR. E-mail: menosso@cnpso.embrapa.br

${ }^{3}$ Eng. Agrôn., Faculdade de Agronomia, UFRGS, Caixa Postal 776, CEP 90001-970 Porto Alegre, RS. E-mail: jamc@vortex.ufrgs.br, ibanghi@vortex.ufrgs.br, bohnen@conex.com.br
}

e de acidez não tem sido suficientemente estudado no Brasil. A acidez dos solos, com $\mathrm{Al}$ em níveis tóxicos e baixa disponibilidade de elementos essenciais, exige aplicações de calcário e fertilizantes para a sua adequada utilização agrícola. A possibilidade de aproveitar a capacidade que as plantas têm de se adaptar a diferentes ecossistemas agrícolas é ampla, decorrente de fatores como os de ordem econômica, da utilização de áreas marginais e da estabilidade de produção. Assim, a identificação da tolerância à toxicidade do Al nos genótipos de soja, torna-se necessária, tendo em vista, também, as grandes diferenças socioeconômicas dos agricultores. 
A utilização de solução nutritiva para avaliação da tolerância de plantas ao $\mathrm{Al}$ tem sido comum, por constituir um meio rápido e bastante eficiente, com a possibilidade de avaliação e seleção de um grande número de genótipos em pouco tempo. A vantagem é permitir a observação dos efeitos do $\mathrm{Al}$ sobre as raízes em fases iniciais, sem que seja interrompido o desenvolvimento das plantas (Moore et al., 1976). Considerando que o efeito primário do $\mathrm{Al}$ é sobre as raízes, com inibição da divisão celular, o parâmetro mais utilizado é o do desenvolvimento das raízes, com ênfase no comprimento da raiz principal (Hanson \& Kamprath, 1979; Sapra et al., 1982; Mascarenhas et al., 1984a, 1984b).

Soluções com nível fixo de Ca e níveis variáveis de $\mathrm{Al}$ têm sido usadas para determinar a tolerância de plantas a esse elemento (Sartain \& Kamprath, 1978; Hanson \& Kamprath, 1979; Fonseca Júnior et al., 1981). A ausência de P, nessas soluções, é embasada nas observações de que $90 \%$ do Al é precipitado com o uso de $10 \mathrm{mg} \mathrm{L}^{-1}$ de $\mathrm{P}$ em $\mathrm{pH}$ entre 4,0 e 4,5 (Munns, 1965), e que as necessidades dos nutrientes das plântulas de soja durante os primeiros dias após a emergência são atendidas pela translocação dos cotilédones (McAlister \& Krober, 1951). Isso sugere que, em ensaios de curta duração, os elementos essenciais, exceto o $\mathrm{Ca}$, podem ser dispensados.

A necessidade de utilização de Ca na solução devese ao fato de que a sua translocação ascendente na planta, via xilema, é feita aparentemente sem restrições. No entanto, o movimento descendente praticamente inexiste (Marschner \& Richter, 1974). Sabese que o Ca influencia o grau de fitotoxicidade do Al: aumentando o suprimento de $\mathrm{Ca}$, é reduzida a absorção de Al (Munns, 1965; Lance \& Pearson, 1969) e, aumentando o suprimento de Al, causa redução na absorção de Ca (Munns, 1965). Mesmo utilizando somente $\mathrm{Ca}$ e $\mathrm{Al}$, não existe consenso quanto aos respectíveis níveis, em decorrência de fatores como a temperatura, tempo de duração do ensaio, controle de $\mathrm{pH}$ e interação entre nutrientes (Sartain \& Kamprath, 1978; Hanson \& Kamprath, 1979; Fonseca Júnior et al., 1981).

Uma série de experimentos foi, então, conduzida com os objetivos de a) determinar os níveis de $\mathrm{Al} \mathrm{e}$ Ca em solução diluída e do melhor período de de- senvolvimento das raízes para a discriminação dos genótipos de soja em relação à tolerância ao $\mathrm{Al}$; b) identificar e classificar 148 genótipos de soja quanto à toxicidade de $\mathrm{Al}$; c) avaliar a capacidade de absorção de $\mathrm{Al}$ e Ca por diferentes genótipos de soja.

\section{MATERIAL E MÉTODOS}

\section{Ensaios de definição de metodologia}

Foram realizados três ensaios em solução de baixa concentração de sais (solução diluída), contendo doses variáveis de $\mathrm{Al}$ e doses fixas de $\mathrm{Ca}$, diferentes em cada ensaio. No primeiro ensaio, a dose de Ca foi de $30 \mathrm{mg} \mathrm{L}^{-1}$, como $\mathrm{CaCl}_{2} \cdot 2 \mathrm{H}_{2} \mathrm{O}$, e as doses de $\mathrm{Al}$ foram: $0,0,1,0,2,0 \mathrm{e}$ $6,0 \mathrm{mg} \mathrm{L}^{-1}$, como $\mathrm{Al}_{2}\left(\mathrm{SO}_{4}\right)_{3} \cdot 18 \mathrm{H}_{2} \mathrm{O}$. No segundo ensaio, a dose de Ca foi de $50 \mathrm{mg} \mathrm{L}^{-1}$, e as doses de $\mathrm{Al}$ foram: 0,0, $0,5,1,0$ e $3,0 \mathrm{mg} \mathrm{L}^{-1}$. No terceiro ensaio, a dose de Ca foi a mesma do segundo ensaio, e as de $\mathrm{Al}$ foram 0,0, 0,1, 0,2 e $0,4 \mathrm{mg} \mathrm{L}^{-1}$. $\mathrm{O} \mathrm{pH}$ da solução foi inicialmente ajustado para $4,5 \mathrm{com} \mathrm{HCl} 0,5 \mathrm{~N}$, e não foi mais ajustado até o final do ensaio. Foram utilizados recipientes de plástico azul-escuro, com dimensões de $61 \times 41 \times 18 \mathrm{~cm}$ e capacidade de 36 litros. Para suportar as plantas, foram utilizadas placas de isopor de $60 \times 40 \mathrm{~cm}$, com $2 \mathrm{~cm}$ de espessura, com 60 furos de $1,9 \mathrm{~cm}$ de diâmetro. Cada planta foi sustentada por um cilindro de isopor com o diâmetro do furo da placa, cortado longitudinalmente até $3 / 4$ do diâmetro. $\mathrm{O}$ arejamento da solução foi feito através de borbulhamento de ar, de modo contínuo, suprido por um moto-compressor. No primeiro e segundo ensaio, foram utilizadas quatro cultivares de soja, FT-1, FT-2, IAC-7, IAC-9 e seis linhagens provenientes de seleção em solo ácido, com 54\% de saturação de Al, nomeadas como BRAS83-1488, BRAS83-1740, BRAS84-2925, BRAS85-1760, BRAS86-3931 e BRAS86-4460 (Menosso et al., 1988). No terceiro ensaio, foram utilizados dez genótipos de soja; Andrews, BR-4, FT-1, FT-19, IAC-4, Paraná, Paranagoiana, Pérola, Santa Rosa e BRAS86-4460. As sementes foram previamente germinadas em câmara, e as plântulas foram transplantadas no quarto dia, sendo escolhidas as que apresentaram as raízes mais uniformes. As medidas do comprimento da raiz primária, do colo da planta até a extremidade da coifa, foram efetuadas inicialmente no transplante e após dez dias ( $1^{\circ}$ ensaio), oito dias ( $2^{\circ}$ ensaio) e três, seis e nove dias ( $3^{\circ}$ ensaio). O incremento foi verificado por diferença, entre as datas de amostragem. Foi utilizado o valor médio de seis plantas para cada genótipo, por tratamento. Os ensaios foram conduzidos em casa de vegetação do Departamento de Solos da Universi- 
dade Federal do Rio Grande do Sul, sem controle de luminosidade e temperatura. Foi utilizado o delineamento inteiramente casualizado no primeiro e segundo ensaio, e o de blocos casualizados, no terceiro ensaio, com os tratamento distribuídos em esquema fatorial $4 \times 3 \times 10$, com três blocos, sendo o primeiro fator as concentrações de $\mathrm{Al}$, o segundo, as épocas de anotações, e o terceiro, os genótipos de soja. Em razão de haver crescimento de raízes muito reduzido nos níveis mais elevados de $\mathrm{Al}$ nos dois primeiros ensaios, as observações foram transformadas segundo $(x+1)^{1 / 2}$ para a execução da análise de variância nesses ensaios.

\section{Ensaios de avaliação de genótipos}

Foi conduzida uma série de 15 ensaios, em seqüência, para a avaliação de 148 genótipos de soja, compostos por cultivares comerciais brasileiras, linhagens de interesse e cultivares-padrões norte-americanas de reação contrastante ao $\mathrm{Al}$ (Tabela 1). Nas soluções, foi utilizada a concentração fixa de Ca de $50 \mathrm{mg} \mathrm{L}^{-1} \mathrm{e}$ os níveis de $\mathrm{Al}$ de $0,0 \mathrm{e}$ $0,2 \mathrm{~m} \mathrm{~L}^{-1}$, sendo esta identificada, nos ensaios de definição de metodologia, como a dose que separa a tolerância de genótipos ao Al. $\mathrm{O}$ pH inicial da solução foi ajustado para 4,5, com $\mathrm{HCl} 0,5 \mathrm{~N}$ e não mais ajustado até o final dos ensaios. As medidas de comprimento da raiz primária, no início e no término do ensaio forneceram, por diferença, o incremento radicular. Os ensaios foram conduzidos por nove dias. O local, a metodologia e a estrutura dos ensaios foram os mesmos dos ensaios de definição de metodologia. O delineamento foi de blocos casualizados com os tratamentos distribuídos em esquema fatorial de $2 \times 10$, com três blocos, sendo o primeiro fator as concentrações de Al, e o segundo, os genótipos de soja.

Para a classificação dos genótipos de soja em relação à sua tolerância ao Al, foi utilizada a Análise de Agrupamento (Cluster Analysis) realizada pelo programa de computação SPSS - Statistical Package for the Social Sciences (Norusis, 1983). Na comparação entre os critérios de classificação da tolerância ao $\mathrm{Al}$, foram usados os dados de incremento verificados na raiz primária na concentração e $0,2 \mathrm{mg} \mathrm{L}^{-1}$ de Al. A técnica consiste em transformar os dados obtidos em uma matriz de similaridade. O cálculo dos coeficientes, que medem a distância entre dois genótipos em um conjunto de n-variáveis comuns, foi realizado através da utilização da Distância Euclidiana ao Quadrado. A Análise de Agrupamento foi realizada pelo Método de Ward, e o conglomerado dos genótipos formado foi representado pelo dendrograma classificatório ou árvore gráfica (Hair Junior et al., 1987).

\section{Ensaio de avaliação da absorção de $\mathrm{Al}$ e Ca por genótipos}

Foi conduzido um ensaio em solução contendo as concentrações de $50 \mathrm{mg} \mathrm{L}^{-1}$ de Ca e $0,0,0,1,0,2$ e $0,4 \mathrm{mg} \mathrm{L}^{-1}$ de Al, para avaliação do efeito recíproco na absorção desses elementos por genótipos de soja. $\mathrm{O} \mathrm{pH}$ inicial foi de 4,5, ajustado com $\mathrm{HCl}$ 0,5 N. Foram usados os mesmos recipientes e metodologia descritos anteriormente. Foram utilizados oito genótipos de soja, divididos em dois grupos em função da reação ao Al. Os genótipos do grupo tolerante ao Al foram Bragg, EMGOPA-304 (Campeira), FT-1 e FT-6 (Veneza), e os do grupo sensível foram EMGOPA-303, IAC-13, Paraná e SPS-1 (COPERSUCAR 1). Foi utilizado o delineamento de blocos casualizados, em esquema fatorial de $4 \times 8$, com três blocos. O ensaio desenvolveu-se em casa de vegetação, sem controle de luminosidade e temperatura, por nove dias.

Após a colheita das plantas, as raízes foram separadas da parte aérea e colocadas em estufa, com ar forçado a $60^{\circ} \mathrm{C}$, até peso constante. As determinações do Ca e do $\mathrm{Al}$ nas amostras secas seguiram a metodologia descrita por Greweling (1976).

\section{RESULTADOS E DISCUSSÃO}

\section{Metodologia para a separação dos genótipos de soja em solução nutritiva diluída}

As raízes dos genótipos de soja apresentaram-se atrofiadas e limitadas à raiz primária em qualquer dos níveis de $\mathrm{Al}$ adicionados à solução no primeiro $\mathrm{e}$ segundo ensaio (Tabelas 2 e 3 ). As concentrações de 1,0, 2,0 e 6,0 $\mathrm{mg} \mathrm{L}^{-1}$ de Al (Tabela 2) e de $1,0 \mathrm{e}$ $3,0 \mathrm{mg} \mathrm{L}^{-1}$ de $\mathrm{Al}$ (Tabela 3 ) não determinaram diferenças $(\mathrm{P}<0,05)$ entre os efeitos médios dos genótipos de soja em qualquer das concentrações de $\mathrm{Ca}$ (30 ou $\left.50 \mathrm{mg} \mathrm{L}^{-1}\right)$. As diferenças entre os genótipos foram verificadas na concentração de $0,5 \mathrm{mg} \mathrm{L}^{-1}$ de Al e de $50 \mathrm{mg} \mathrm{L}^{-1} \mathrm{de} \mathrm{Ca}$, na qual a linhagem BRAS86-3931 e a cultivar FT-1 foram as menos sensíveis (Tabela 3). As reduções no comprimento da raiz primária atingiram médias de $93 \%$ e $92 \%$, respectivamente, nesses ensaios (Tabelas 2 e 3), demonstrando que esses níveis de Al são muito altos para separar os genótipos quanto à tolerância. Alguns autores (Sartain \& Kamprath,1978; Fonseca Júnior et al., 1981) também não obtiveram separação na tolerância entre genótipos de soja nos níveis de $\mathrm{Al}$ de 1,0, 2,5 e $3,5 \mathrm{mg} \mathrm{L}^{-1}$. 
TABELA 1. Genealogia dos genótipos de soja utilizados nos ensaios.

\begin{tabular}{|c|c|c|c|}
\hline Genótipo & Genealogia & Genótipo & Genealogia \\
\hline Andrews & Seleção em Santa Rosa & FT-7 (Tarobá) & FT-4 x Davis \\
\hline BABR-31 & UFV-1 X M-4-1 & FT-8 (Araucária) & Cobb x Planalto \\
\hline Biloxi & PI 23.211 (Tsze Pi Tou -China) & FT-9 (Inaê) & FT-4 x Davis \\
\hline Bossier & Mutação natural em Lee & FT-10 (Princesa) & FT9510 x Sant'Ana \\
\hline Bragg & Jackson x D49-2491 & FT-11 (Alvorada) & UFV-1 x Campos Gerais \\
\hline BRAS83-1488 & Davis $x$ BR-5 & FT-12 (Nissei) & FT9510 x Prata \\
\hline BRAS83-1574 & Lancer x União & FT-13 (Aliança) & Davis x FT 216 \\
\hline BRAS83-1740 & Bragg x PI346.304 & FT-14 (Piracema) & FT9510 x Sant'Ana \\
\hline BRAS84-2925 & Paraná x BR-1 & FT-15 & FT9510 x Sant'Ana \\
\hline BRAS85-1736 & Davis x Paraná & FT-16 & FT440 x Campos Gerais \\
\hline BRAS85-1760 & Davis x Paraná & FT-17 (Bandeirante) & Seleção em FT-2 \\
\hline BRAS85-1824 & Davis $\mathrm{x}$ IAS 4 & FT-18 (Xavante) & FT9510 x Prata \\
\hline BRAS86-3672 & FT79-3637 x (Perry x C. Gerais) & FT-19 (Macacha) & Santa Rosa x (sel. em Cajeme x São Luíz) \\
\hline BRAS86-3701 & Lancer x PI346.304 & FT-20 (Jaú) & FT-4 x Davis \\
\hline BRAS86-3931 & OCEPAR 2 = Iapó $\mathrm{x}$ Perry & Garimpo (MGBR-22) & Bossier x Paraná \\
\hline BRAS86-4460 & BR-1 x (União x Biloxi) & GOBR-25 (Aruanã) & E77-510-3 x BR78-11202 \\
\hline BR EMGOPA-312 (Potiguar) & Paranagoiana x Cristalina & GOBR-26 (Tocantins)PR & Paraná x PR77-10001 \\
\hline BR-1 & Hill x L356 (=Industrial) & Hardee & D49-772 x Improved Pelican \\
\hline BR-2 & Hill $x$ Hood & IAC-4 & IAC- 2 x Hardee \\
\hline BR-3 & Hampton x Campos Gerais & IAC-5 & Seleção na população FB59-1 \\
\hline BR-4 & Hill x Hood & IAC-6 & Seleção na população RB72-1 \\
\hline BR-6 (Nova Bragg) & Bragg (3) x Santa Rosa & IAC-7 & Seleção na população RB72-1 \\
\hline BR-7 & Hardee x Hill & IAC- 8 & Bragg x (Hill x PI240.664) \\
\hline BR-8 (Pelotas) & Bienville $\mathrm{x}$ Hampton & IAC-9 & Seleção na população RB72-1 \\
\hline BR-9 (Savana) & Seleção na população LoB74-2 & IAC- 12 & Paraná x IAC73-231 \\
\hline BR-10 (Teresina) & UFV-1 x IAC73-2736-10 & IAC-13 & Paraná x IAC73-231 \\
\hline BR-12 & Bienville $x$ Hood & IAC-17 & Seleção na população B-5 \\
\hline BR-13 (Maravilha) & Bragg (4) x Santa Rosa & IAC- 100 & IAC78-2318 x IAC-12 \\
\hline BR-14 (Modelo) & Santa Rosa x Campos Gerais & IAC-Foscarin 31 & Seleção em Foscarin \\
\hline BR-15 (Mato-Grosso) & Santa Rosa x LoD76-761 & IAS 3-Delta & Ogden $x$ CNS \\
\hline BR-16 & D69-B10xM58 x Davis & IAS 4 & Hood $x$ Jackson \\
\hline BR-23 & Bossier x Paraná & IAS 5 & Hill x D52-810 \\
\hline BR-24 & Davis x Paraná & Invicta & Lancer $x$ Essex \\
\hline BR-27 (Cariri) & BR78-22043 x (Bragg x IAC73-2736) & IPAGRO-20 & (Santa Rosa x Arksoy) x (Majos x Kanro) \\
\hline BR-28 (Seridó) & Santa Rosa x BR78-11202 & IPAGRO-21 & (Forest $\mathrm{x}$ Hood) $\mathrm{x}$ Lousiania \\
\hline BR-29 (Londrina) & Davis $x$ BR- 5 & Ivaí & Majos x Hood \\
\hline BR-30 & União (2) x Lo76-1763 & Ivorá & (Davis $\mathrm{x}$ Shinan.) $\mathrm{x}$ (Hogioku $\mathrm{x}$ A.Com.) \\
\hline BR-36 & IAS 4 (2) x BR78-22043 & $\mathrm{J}-200$ & IAC- 2 x Viçoja \\
\hline BR-37 & União (2) x Lo 76-1763 & Lancer & Paraná x Hampton 266 \\
\hline BR-38 & FT-2 x União & LC72-749 & Bienville $\mathrm{x}$ Hood \\
\hline BR-40 (Itiquira) & Davis (2) x (Hill x PI240.664) & Lee 68 & Lee(6) x Arksoy \\
\hline Campos Gerais & Arksoy x Ogden & Majos & Tokio $\mathrm{x}$ Yelredo \\
\hline Chief & Illini x Manchu A (T95) & MSBR-17 (São Gabriel) & Lo76-732 x LoD76-736 \\
\hline Cobb & F57-735 x D58-3358 & MSBR-18 (Guavira) & Cruzamento natural em Viçoja \\
\hline Cristalina & Cruzamento natural em UFV-1 & MSBR-19 (Pequi) & D69-442 x (Bragg x Santa Rosa) \\
\hline Davis & (Roan. x [Ogden x CNS])x(Ral. x Ogden) & MSBR-20 (Ipê) & D69-6344 x (Bragg x Santa Rosa) \\
\hline EMGOPA-301 & IAC-4 x Júpiter & MSBR-21 (Buriti) & São Luiz x Davis-1 \\
\hline EMGOPA-302 & Paraná x Mandarin & MSBR-39 (Chapadão) & Doko x M-4-1 \\
\hline EMGOPA-303 & IAC73-2736-10 x IAC-6 & Nova IAC-7 & Seleção em IAC-7 \\
\hline EMGOPA-304 (Campeira) & Paraná x Mandarin & Numbaíra I & Davis $x$ Kedelle STB n 452 \\
\hline EMGOPA-305 (Caraíba) & Tropical x Cristalina & OCEPAR 2 = Iapó & Hampton $208 \times$ Davis \\
\hline EMGOPA-306 (Chapada) & Seleção em EMGOPA-301 & OCEPAR $3=$ Primavera & (Halesoy x Volstate) x (Hood x Rhosa) \\
\hline EMGOPA-307 (Caiapó) & GO79-3090 x Paranagoiana & OCEPAR 5 = Piquiri & Coker 136 x Co $72-260$ \\
\hline EMGOPA-308 (S. Dourada) & Seleção em EMGOPA-301 & OCEPAR 6 & (PI230.979xLee68) $\mathrm{x}$ (Davis $\mathrm{x}$ Bragg) $\mathrm{x}$ (Dare $\mathrm{x}$ Davis) $]$ \\
\hline EMGOPA-309 (Goiana) & Seleção em BRB-214 & OCEPAR 7 = Brilhante & Cruzamento natural em IAS 5 \\
\hline EMGOPA-310 & Seleção em BRB-256 & OCEPAR 8 & Cruzamento natural em Paraná \\
\hline EMGOPA-311 & Paranagoiana $\mathrm{x}$ Cristalina & OCEPAR $9=$ SS 1 & Mutação natural em Paraná \\
\hline EMGOPA-313 & IAC-7 x (Santa Rosa x GO79-3068) & OCEPAR 10 & Paraná x União \\
\hline FT-Abyara & União x Sant'Ana & OCEPAR 11 & Davis x Paraná \\
\hline FT-Bahia & Cruzamento natural em Cristalina & Paraná & Hill x D52-810 \\
\hline FT-Canarana & Cristalina x FT-1 & Paranagoiana & Mutação natural em Paraná \\
\hline FT-Cometa & FT420 x Williams & Paranaíba & Davis x IAC72-2211 \\
\hline FT-Estrela & M- 2 x FT-1 & Pérola & Hood x Industrial \\
\hline FT-Eureka & Paraná x (PI346.304 x Paraná) & Perry & Patoka x L37-1355(=PI81.041) \\
\hline FT-Guaíra & Lancer x União & Planalto & Hood $x$ Kedelle STB $n^{\circ} 452$ \\
\hline FT-Jatobá & FT9510 x Sant'Ana & RS 7-Jacuí & Ivorá x PI80.837 \\
\hline FT-Manacá & FT907 x Lancer & Santa Rosa & D49-772 x La41-1219 \\
\hline FT-Maracajú & FT9510 x Sant'Ana & Sertaneja & Paraná x Hampton 266 \\
\hline FT-Seriema & M- 2 x FT- 1 & SPBR-41 & Co136 x Paranaíba \\
\hline FT-1 & Seleção em Sant'Ana & SPS-1 (COPERSUCAR 1) & Lancer x Paranaíba \\
\hline FT-2 & Seleção em IAS 5 & Tiarajú & Industrial x Asomusume \\
\hline FT-3 & Cruzamento natural em Flórida & Timbira & Seleção na população RB72-1 \\
\hline FT-4 & D65-3075 x D64-4636 & Tropical & Hampton x E70-51 \\
\hline FT-5 (Formosa) & FT9510 x Sant'Ana & UFV-1 & Seleção em Viçoja \\
\hline FT-6 (Veneza) & FT9510 x Prata & UFV-4 & IAC- 2 x Mineira \\
\hline
\end{tabular}


As interações tríplices, obtidas no terceiro ensaio, mostraram diferenças $(\mathrm{P}<0,05)$ nas leituras do sexto e do nono dia, não havendo a possibilidade de separação na tolerância entre os genótipos na leitura do terceiro dia (Tabela 4). A separação da reação dos genótipos em relação aos níveis de $\mathrm{Al}$ ficou melhor caracterizada na leitura do nono dia nas concentrações de 0,1 e $0,2 \mathrm{mg} \mathrm{L}^{-1}$ de Al. Na avaliação geral, a

TABELA 2. Comprimento $(\mathrm{cm})$ da raiz primária de genótipos de soja em solução diluída (30 $\mathrm{mg} \mathrm{L}^{-1}$ de $\mathrm{Ca}$ ) com diferentes níveis de alumínio'.

\begin{tabular}{lllll}
\hline \multirow{2}{*}{ Genótipo } & \multicolumn{4}{c}{ Nível de alumínio $\left(\mathrm{mg} \mathrm{L}^{-1}\right)$} \\
\cline { 2 - 5 } & 0,0 & 1,0 & 2,0 & 6,0 \\
\hline FT-1 & $26,0 \mathrm{bcd}$ & $0,4 \mathrm{a}$ & $0,5 \mathrm{a}$ & $0,4 \mathrm{a}$ \\
FT-2 & $25,3 \mathrm{~cd}$ & $0,2 \mathrm{a}$ & $0,5 \mathrm{a}$ & $0,3 \mathrm{a}$ \\
IAC-7 & $26,3 \mathrm{bcd}$ & $0,7 \mathrm{a}$ & $0,6 \mathrm{a}$ & $0,5 \mathrm{a}$ \\
IAC-9 & $27,7 \mathrm{abc}$ & $0,8 \mathrm{a}$ & $0,5 \mathrm{a}$ & $0,6 \mathrm{a}$ \\
BRAS83-1488 & $25,2 \mathrm{~cd}$ & $0,5 \mathrm{a}$ & $0,7 \mathrm{a}$ & $0,6 \mathrm{a}$ \\
BRAS83-1740 & $23,5 \mathrm{~d}$ & $0,9 \mathrm{a}$ & $0,9 \mathrm{a}$ & $0,6 \mathrm{a}$ \\
BRAS84-2925 & $31,3 \mathrm{a}$ & $0,6 \mathrm{a}$ & $0,5 \mathrm{a}$ & $0,4 \mathrm{a}$ \\
BRAS85-1760 & $29,3 \mathrm{ab}$ & $0,3 \mathrm{a}$ & $0,6 \mathrm{a}$ & $0,5 \mathrm{a}$ \\
BRAS86-3931 & $28,0 \mathrm{abc}$ & $0,9 \mathrm{a}$ & $1,3 \mathrm{a}$ & $0,4 \mathrm{a}$ \\
BRAS86-4460 & $19,8 \mathrm{e}$ & $0,5 \mathrm{a}$ & $0,7 \mathrm{a}$ & $0,3 \mathrm{a}$ \\
\hline \multicolumn{5}{c}{${ }^{1}$ Valores seguidos de mesma letra na coluna não diferem entre si pelo teste } \\
de Tukey, a 5\% de probabilidade.
\end{tabular}

TABELA 3. Crescimento $(\mathrm{cm})$ da raiz primária de genótipos de soja em solução diluída (50 $\mathrm{mg} \mathrm{L}^{-1}$ de Ca) com diferentes níveis de alumínio'.

\begin{tabular}{lllll}
\hline Genótipo & \multicolumn{4}{c}{ Nível de alumínio $\left(\mathrm{mg} \mathrm{L}^{-1} \mathrm{de} \mathrm{Ca}\right)$} \\
\cline { 2 - 5 } & 0,0 & 0,5 & 1,0 & 3,0 \\
\hline FT-1 & $39,0 \mathrm{a}$ & $6,5 \mathrm{a}$ & $0,9 \mathrm{a}$ & $0,3 \mathrm{a}$ \\
FT-2 & $34,1 \mathrm{abc}$ & $2,6 \mathrm{~b}$ & $0,6 \mathrm{a}$ & $0,3 \mathrm{a}$ \\
IAC-7 & $37,3 \mathrm{ab}$ & $2,0 \mathrm{~b}$ & $0,6 \mathrm{a}$ & $0,3 \mathrm{a}$ \\
IAC-9 & $30,6 \mathrm{bcd}$ & $1,5 \mathrm{~b}$ & $0,7 \mathrm{a}$ & $0,5 \mathrm{a}$ \\
BRAS83-1488 & $28,0 \mathrm{~d}$ & $2,2 \mathrm{~b}$ & $0,7 \mathrm{a}$ & $0,4 \mathrm{a}$ \\
BRAS83-1740 & $27,6 \mathrm{~d}$ & $3,4 \mathrm{~b}$ & $0,4 \mathrm{a}$ & $0,3 \mathrm{a}$ \\
BRAS84-2925 & $36,1 \mathrm{abc}$ & $2,3 \mathrm{~b}$ & $0,7 \mathrm{a}$ & $0,4 \mathrm{a}$ \\
BRAS85-1760 & $34,5 \mathrm{abcd}$ & $1,7 \mathrm{~b}$ & $1,0 \mathrm{a}$ & $0,3 \mathrm{a}$ \\
BRAS86-3931 & $39,9 \mathrm{a}$ & $8,2 \mathrm{a}$ & $1,6 \mathrm{a}$ & $0,6 \mathrm{a}$ \\
BRAS86-4460 & $29,5 \mathrm{~cd}$ & $1,9 \mathrm{~b}$ & $0,4 \mathrm{a}$ & $0,2 \mathrm{a}$ \\
\hline 1 Valores seguidos de mesma letra na coluna não diferem entre si pelo teste \\
de Tukey, a 5\% de probabilidade.
\end{tabular}

concentração de $0,2 \mathrm{mg} \mathrm{L}^{-1}$ de $\mathrm{Al}$ mostrou maior eficiência na separação da tolerância entre os genótipos. A concentração de $0,4 \mathrm{mg} \mathrm{L}^{-1}$ de $\mathrm{Al}$ não permitiu essa separação, em nenhuma das leituras. Se observada a leitura do sexto dia, as cultivares FT-1, Andrews, Pérola e Santa Rosa, embora fossem as mais tolerantes, não puderam ser diferenciadas entre si. No entanto, a expressão de classes de tolerância foi melhor caracterizada na avaliação do nono dia: a FT-1 como a mais tolerante, seguida da Andrews. Entretanto, alguns autores conseguiram boa separação na tolerância ao Al entre os genótipos de soja no quinto dia (Hanson \& Kamprath, 1979), no sexto dia (Sapra et al., 1982) e, no sétimo dia (Mascarenhas et al., 1984a), em virtude da utilização de diferentes soluções, parâmetros de avaliação e tipo de controle dos fatores ambientais.

\section{Avaliação de cultivares e linhagens brasileiras de soja}

Os resultados dos ensaios de avaliação apresentaram interação entre os genótipos de soja e níveis de Al. Mediante o incremento da raiz primária, na concentração de $0,2 \mathrm{mg} \mathrm{L}^{-1} \mathrm{de} \mathrm{Al}$, foram obtidas diferenças $(\mathrm{P}<0,05)$ entre os efeitos médios dos genótipos em todos os ensaios realizados. A avaliação conjunta dos ensaios utilizando a Análise de Agrupamento, pelo Método de Ward, permitiu a análise grupal de ensaios, identificando as classes de tolerância entre os genótipos avaliados. Esta análise compensa o fato de que, em cada ensaio, foram utilizados genótipos diferentes, não necessitando de padrões de sensibilidade (Hair Junior et al., 1987). O dendrograma, com a análise aglomerativa dos genótipos de soja, encontra-se na Fig. 1. Este dendrograma foi dividido em três grupos que classificam os genótipos de soja em: A) grupo de genótipos tolerantes; B) grupo de genótipos de tolerância intermediária; e C) grupo de genótipos sensíveis.

$\mathrm{O}$ grupo A, que compreende os genótipos classificados como tolerantes ao Al, foi constituído por 21 cultivares e linhagens de soja. Neste grupo, há duas condições. Na primeira, todos os genótipos têm em um dos pais o caráter de tolerância ao $\mathrm{Al}$, envolvido no cruzamento. Assim, a Bragg tem a cultivar Jackson, como pai tolerante; a EMGOPA-302 e 


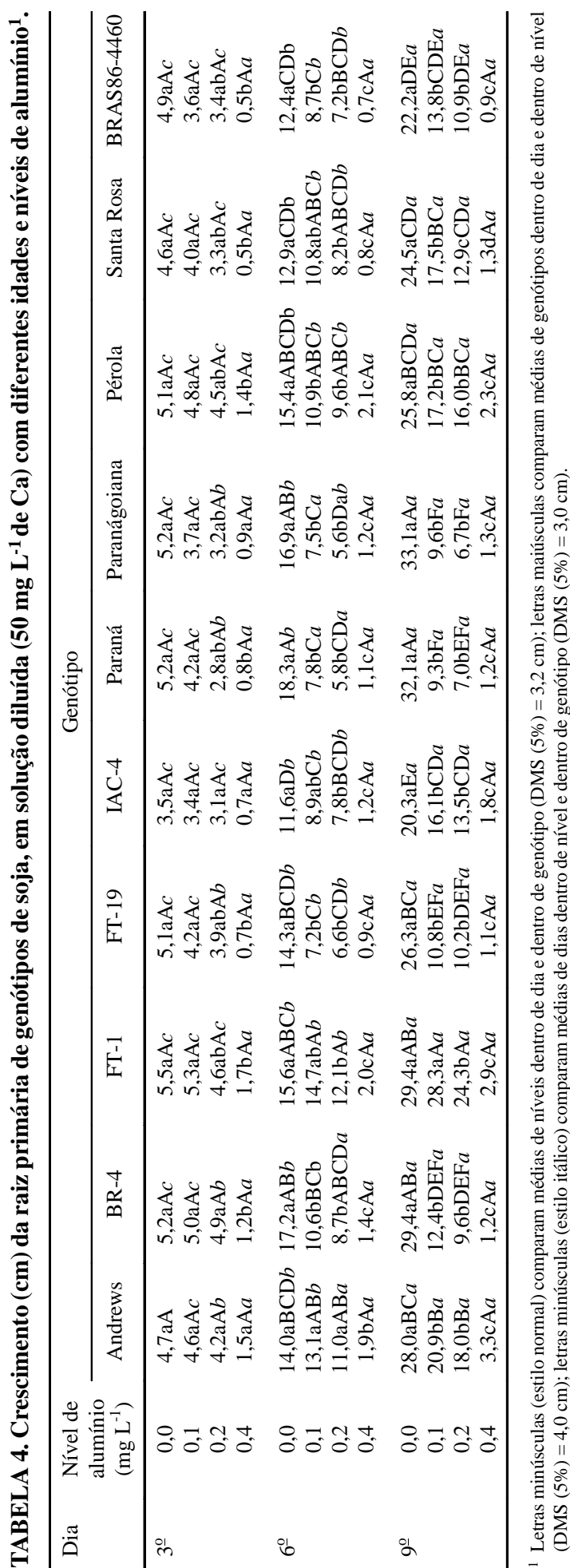

EMGOPA-304 têm a cultivar Mandarin, proveniente da China, tolerante (Armiger et al., 1968); a BR-13 tem a Bragg como pai tolerante, em retrocruzamento por quatro vezes. Linhas derivadas de retrocruzamentos, desenvolvidas sem seleção para tolerância ao Al, tiveram o mesmo comportamento de tolerância quanto ao pai recorrente (Reid et al., 1969). A FT-1, proveniente de seleção em Sant'Ana, linhairmã de Lee, tolerante (Armiger et al., 1968; Sartain \& Kamprath, 1978); e a Planalto, proveniente de Hood, que é tolerante (Sartain \& Kamprath, 1978). Na outra condição, ambos os pais são tolerantes, como na IAS 4, proveniente de Hood x Jackson (Armiger et al., 1968; Sartain \& Kamprath, 1978). O caráter tolerância deve estar distribuído nos ancestrais, provenientes dos centros de origem da soja, ou nos parentais próximos. Não há genótipo essencialmente tolerante, e a tolerância verificada nos genótipos avaliados é casual, por não haver pesquisa específica, com seleção em meio com Al.

O grupo B, de tolerância intermediária, foi composto por 73 genótipos. As linhagens identificadas pela sigla BRAS $(\mathrm{BR}=$ Brasil e $\mathrm{AS}=$ Acidez do Solo) foram selecionadas em solo ácido, com 54\% de saturação de $\mathrm{Al}$ (Menosso et al., 1988) e classificadas, na sua maioria, neste grupo. A seleção em solo ácido não condiciona, necessariamente, tolerância ao Al. O solo é um sistema tamponado e não mantém altos teores de $\mathrm{Al}$ e baixos de manganês ou vice-versa (Neenan, 1960). Os genótipos norte-americanos Perry \& Lee 68, identificados como tolerantes por Armiger et al. (1968), foram classificados como de tolerância intermediária, comprovando a existência de genótipos com tolerância em maior grau ao $\mathrm{Al}$ em relação aos padrões norte-americanos (Sapra et al., 1982). O grupo de tolerância intermediária ao $\mathrm{Al}$ foi constituído pelo maior número de genótipos, seguido pelo grupo de genótipos sensíveis (Fig. 1). A tolerância intermediária é dada em razão de que um dos pais, ou os ancestrais, apresentam determinada tolerância, cujo processo de seleção não foi feito em condições de alta saturação de $\mathrm{Al}$ e, portanto, se houve tolerância intermediária, ela é casual. Fonseca Júnior (1982) estudou o tipo de herança e a herdabilidade da tolerância à toxicidade por $\mathrm{Al}$ em soja, e constatou que houve uma segregação irregular, na geração $\mathrm{F}_{2}$, não seguindo o padrão caracterís- 
A-tolerante

B-intermediário

C-sensível

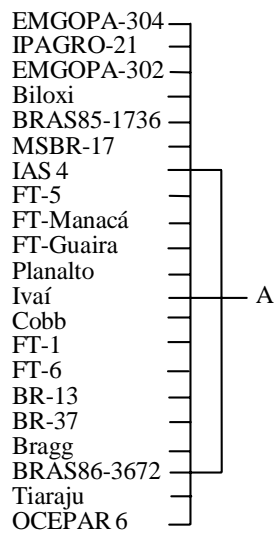

BR-1

Davis

FT-14

IAC-9

FT-16

FT-9

BRAS86-4460

BABR-31

BR-7

BRAS83-1740

BR-23

Garimpo

FT-10

BRAS83-1574

IAC-100

Perry

FT-17

OCEPAR 5

Sertaneja

BR-10

BR-27

BR-36

FT-Cometa

IAC-17

MSBR-19

MSBR-18

C.Gerais

BR-14

FT-Eureka

Ivorá

Timbira

FT-3

OCEPAR 2

EMGOPA-308

LC72-749

IAC-4 B

IAC-31

EMGOPA-311

Majos

BR-40

BR-9

Santa Rosa

BR-15

UFV-4

FT-18

BR-29

BRAS85-1760

BR-6

Andrews

FT-12

GOBR-25

IAC-5

OCEPAR 7

Lee 68

BRAS85-1824

Tropical

FT-2

BR-12

EMGOPA-306

$\mathrm{J}-200$

FT-Abyara

FT-13

OCEPAR 3

BR-30

EMGOPA 301

FT-15

Pérola

FT-Maracaju

FT-20

FT-Estrela

IPAGRO-20

FT-8

FT-7

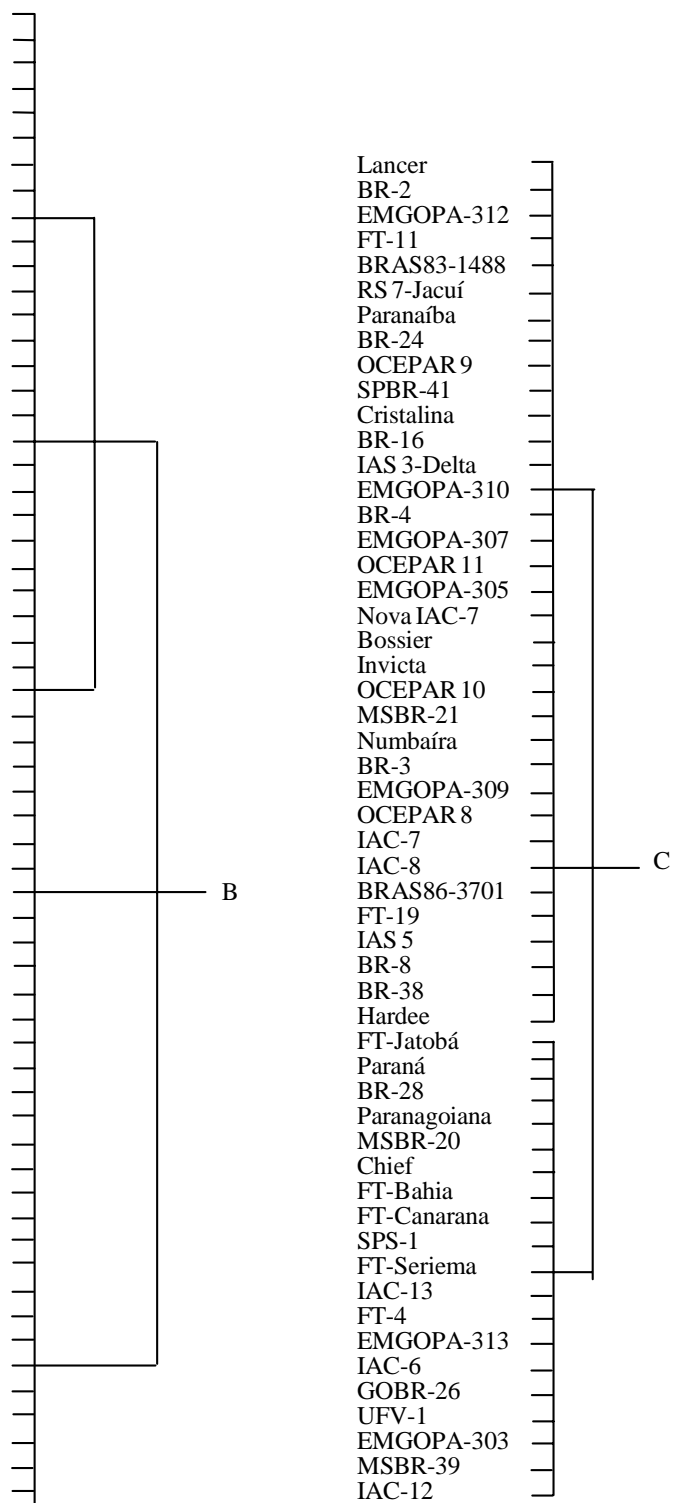

IACBR- 12

FIG.1. Dendrograma da análise de agrupamento (cluster analysis), usando o método de Ward, de 148 genótipos de soja, para o incremento da raiz primária na concentração de $0,2 \mathrm{mg} \mathrm{L}^{-1}$ de alumínio. 
tico de herança monogênica. Apesar dos baixos valores estimados da herdabilidade, tanto no sentido amplo como no sentido restrito, a seleção que o autor realizou foi efetiva. Isto vem comprovar que o caráter de tolerância foi perdido no processo de seleção em condições de solos sem alumínio tóxico e fertilizados a que foram submetidos os genótipos avaliados.

O grupo $\mathrm{C}$, sensível ao $\mathrm{Al}$, foi composto por 54 genótipos. Os genótipos sensíveis ao Al têm, como um dos parentais envolvidos no cruzamento, um genótipo sensível em combinação com outro de tolerância intermediária ou ambos sensíveis. O exemplo clássico de cultivar-padrão de sensibilidade, Chief, identificada por Armiger et al. (1968), provém do cruzamento de Illini x Manchu A (=T95), ambas sensíveis ao Al, segundo Armiger et al. (1968) e Konzak et al. (1976). A presente avaliação também confirmou essa sensibilidade. Os cruzamentos de sensível com sensível produzem sempre genótipos sensíveis, como EMGOPA-313, proveniente de Paranagoiana x Cristalina, ou como Lancer, proveniente de Paraná x Hampton 266 (Sartain \& Kamprath, 1978). No cruzamento de genótipo sensível com genótipo tolerante ou com genótipo de tolerância intermediária, a seleção em solo sem Al tóxico e fertilizado, utilizada normalmente nas instituições de pesquisa brasileiras, não favoreceu a expressão da tolerância ao Al. Assim ocorreu com as cultivares BR-2 e BR-4, ambas provenientes do cruzamento de Hill (sensível) x Hood (tolerante) (Armiger et al., 1968).

\section{Absorção de Al e Ca por genótipos de soja em so- lução nutritiva diluída}

A avaliação do efeito recíproco na absorção de $\mathrm{Al}$ e Ca foi efetuada em ensaio específico envolvendo dois grupos de quatro genótipos, tolerantes e sensíveis à ação do $\mathrm{Al}$, respectivamente. $\mathrm{A}$ presença de $\mathrm{Al}$ na solução afetou, aumentando a concentração desse elemento (Tabela 5) e diminuindo o crescimento radicular (Tabela 6) dos genótipos utilizados. O efeito do $\mathrm{Al}$ foi proporcional às doses aplicadas, e mais intenso nas cultivares sensíveis à sua toxidez.

A exemplo do que ocorreu nos ensaios anteriores, a concentração de $0,4 \mathrm{mg} \mathrm{L}^{-1}$ de $\mathrm{Al}$ foi muito tóxica a todos genótipos testados. A utilização de
$0,2 \mathrm{mg} \mathrm{L}^{-1} \mathrm{de} \mathrm{Al}$, apesar de eficiente para a separação da tolerância dos genótipos com base no crescimento radicular (Tabela 4), não diferenciou os genótipos com base no teor desse elemento nas raízes (Tabela 5). A quantidade de $\mathrm{Al}$ absorvida nessa concentração na solução é, então, determinada pela quantidade de matéria seca produzida pelos genótipos de soja (Tabela 6), que é menor nos sensíveis.

TABELA 5. Concentração de alumínio ( $\left.\mathrm{mg} \mathrm{kg}^{-1}\right)$ no sistema radicular de genótipos de soja desenvolvidos em solução diluída ( $50 \mathrm{mg} \mathrm{L}^{-1}$ de Ca), com diferentes níveis de alumínio ${ }^{1}$.

\begin{tabular}{|c|c|c|c|}
\hline \multirow[t]{2}{*}{ Genótipo } & \multicolumn{3}{|c|}{ Nível de alumínio $\left(\mathrm{mg} \mathrm{L}^{-1}\right)$} \\
\hline & 0,1 & 0,2 & 0,4 \\
\hline \multicolumn{4}{|c|}{ Tolerante ao alumínio } \\
\hline Bragg & $442 \mathrm{aB}$ & $827 \mathrm{aA}$ & $1073 \mathrm{cA}$ \\
\hline EMGOPA-304 & $502 \mathrm{aB}$ & $804 \mathrm{aA}$ & $1.014 \mathrm{cA}$ \\
\hline FT-1 & $459 \mathrm{aC}$ & $734 \mathrm{aB}$ & $1.037 \mathrm{cA}$ \\
\hline FT-6 & $416 \mathrm{aB}$ & $675 \mathrm{aA}$ & $897 \mathrm{cA}$ \\
\hline Média & 455 & 760 & 1.005 \\
\hline \multicolumn{4}{|c|}{ Sensível ao alumínio } \\
\hline EMGOPA-303 & $597 \mathrm{aC}$ & $932 \mathrm{aB}$ & $1.830 \mathrm{aA}$ \\
\hline IAC-13 & $545 \mathrm{aC}$ & $886 \mathrm{aB}$ & 1.667abA \\
\hline Paraná & $675 \mathrm{aB}$ & $886 \mathrm{aA}$ & $1.585 \mathrm{abA}$ \\
\hline SPS-1 & $614 \mathrm{aC}$ & $944 \mathrm{aB}$ & $1.480 \mathrm{bA}$ \\
\hline Média & 608 & 912 & 1.640 \\
\hline
\end{tabular}

TABELA 6. Peso da matéria seca (mg seis plantas $\left.{ }^{-1}\right)$ do sistema radicular de genótipos de soja, desenvolvidos em solução diluída (50 $\mathrm{mg} \mathrm{L}^{-1}$ de Ca) com diferentes níveis de alumínio ${ }^{1}$.

\begin{tabular}{lllll}
\hline \multirow{2}{*}{ Genótipo } & \multicolumn{5}{c}{ Nível de alumínio $\left(\mathrm{mg} \mathrm{L}^{-1}\right)$} \\
\cline { 2 - 5 } & \multicolumn{1}{c}{0,0} & \multicolumn{4}{c}{ Tolerante ao alumínio } \\
\hline Bragg & $0,262 \mathrm{abA}$ & $0,295 \mathrm{aA}$ & $0,215 \mathrm{aA}$ & $0,162 \mathrm{aB}$ \\
EMGOPA-304 & $0,227 \mathrm{bcA}$ & $0,196 \mathrm{bcA}$ & $0,182 \mathrm{abA}$ & $0,125 \mathrm{abB}$ \\
FT-1 & $0,253 \mathrm{abA}$ & $0,260 \mathrm{aA}$ & $0,214 \mathrm{aAB}$ & $0,172 \mathrm{aB}$ \\
FT-6 & $0,295 \mathrm{aA}$ & $0,255 \mathrm{aA}$ & $0,207 \mathrm{aB}$ & $0,159 \mathrm{aC}$ \\
\hline Média & 0,259 & 0,236 & 0,204 & 0,154 \\
\hline & & Sensível ao alumínio & \\
EMGOPA-303 & $0,192 \mathrm{cA}$ & $0,155 \mathrm{cdAB}$ & $0,122 \mathrm{cBC}$ & $0,095 \mathrm{bC}$ \\
IAC-13 & $0,239 \mathrm{abcA}$ & $0,134 \mathrm{~dB}$ & $0,111 \mathrm{cB}$ & $0,090 \mathrm{bB}$ \\
Paraná & $0,230 \mathrm{bcA}$ & $0,149 \mathrm{cdB}$ & $0,115 \mathrm{cBC}$ & $0,097 \mathrm{bC}$ \\
SPS-1 & $0,258 \mathrm{abA}$ & $0,157 \mathrm{cdB}$ & $0,131 \mathrm{bcBC}$ & $0,094 \mathrm{bC}$ \\
\hline Média & 0,230 & 0,149 & 0,120 & 0,094 \\
\hline
\end{tabular}

1 Valores seguidos de mesma letra minúscula, na coluna, e maiúscula na linha, não diferem entre si pelo teste de Tukey, a 5\% de probabilidade. 
Os teores de Ca das raízes (Tabela 7) e da parte aérea (Tabela 8) dos genótipos testados foram afetados pelo $\mathrm{Al}$, especialmente na maior concentração na solução $\left(0,4 \mathrm{mg} \mathrm{L}^{-1}\right)$. Embora os valores de $\mathrm{Ca}$ nas raízes tenham sido majoritariamente mais elevados nos genótipos tolerantes, a discriminação entre os genótipos somente foi possível $(\mathrm{P}<0,05)$ com $0,1 \mathrm{mg} \mathrm{L}^{-1} \mathrm{de} \mathrm{Al}$ (Tabela 7). Na parte aérea, também houve discriminação (Tabela 8), sem, no entanto, ha-

TABELA 7. Conteúdo de cálcio (mg seis plantas $\left.{ }^{-1}\right)$ no sistema radicular de genótipos de soja, desenvolvidos em solução diluída (50 $\mathrm{mg} \mathrm{L}^{-1}$ de Ca) com diferentes níveis de alumínio ${ }^{1}$.

\begin{tabular}{lllll}
\hline Genótipo & \multicolumn{4}{c}{ Nível de alumínio $\left(\mathrm{mg} \mathrm{L}^{-1}\right)$} \\
\cline { 2 - 5 } & \multicolumn{5}{c}{$0,0,1$} & 0,2 & 0,4 \\
\hline \multirow{5}{c}{ Tolerante ao alumínio } \\
Eragg & $1,653 \mathrm{abA}$ & $1,059 \mathrm{abcB}$ & $0,680 \mathrm{aC}$ & $0,454 \mathrm{aC}$ \\
FT-1 & $1,087 \mathrm{dA}$ & $0,897 \mathrm{bcdAB}$ & $0,639 \mathrm{aBC}$ & $0,389 \mathrm{aC}$ \\
FT-6 & $1,321 \mathrm{cdA}$ & $1,202 \mathrm{abA}$ & $0,683 \mathrm{aB}$ & $0,496 \mathrm{aB}$ \\
\hline Média & $1,719 \mathrm{aA}$ & $1,315 \mathrm{aB}$ & $0,747 \mathrm{aC}$ & $0,515 \mathrm{aC}$ \\
\hline \multicolumn{5}{c}{ Sensível ao alumínio } \\
EMGOPA-303 & $1,1445 \mathrm{cdA}$ & $0,774 \mathrm{cdB}$ & $0,558 \mathrm{aBC}$ & $0,361 \mathrm{aC}$ \\
IAC-13 & $1,464 \mathrm{abcA}$ & $0,643 \mathrm{~dB}$ & $0,524 \mathrm{aBC}$ & $0,363 \mathrm{aC}$ \\
Paraná & $1,244 \mathrm{cdA}$ & $0,688 \mathrm{~dB}$ & $0,488 \mathrm{aBC}$ & $0,355 \mathrm{aC}$ \\
SPS-1 & $1,350 \mathrm{bdcA}$ & $0,697 \mathrm{~dB}$ & $0,514 \mathrm{aBC}$ & $0,335 \mathrm{aC}$ \\
\hline Média & 1,307 & 0,700 & 0,521 & 0,354 \\
\hline \multicolumn{5}{c}{ Valores seguidos de mesma letra, minúscula na coluna e maiúscula na } \\
linha, não diferem entre si pelo teste de Tukey, a 5\% de probabilidade.
\end{tabular}

TABELA 8. Conteúdo total de cálcio (mg seis plantas ${ }^{-1}$ ) na parte aérea de genótipos de soja, desenvolvidos em solução diluída $\left(50 \mathrm{mg} \mathrm{L}^{-1} \mathrm{de}\right.$ Ca) com diferentes níveis de alumínio ${ }^{1}$.

\begin{tabular}{lllll}
\hline \multirow{2}{*}{ Genótipo } & \multicolumn{5}{c}{ Nível de alumínio $\left(\mathrm{mg} \mathrm{L}^{-1}\right)$} \\
\cline { 2 - 5 } & \multicolumn{5}{c}{0,0} & 0,1 & 0,2 & 0,4 \\
\hline \multirow{5}{c}{ Tolerante ao alumínio } \\
Bragg & $6,849 \mathrm{abA}$ & $4,386 \mathrm{abB}$ & $3,068 \mathrm{abcC}$ & $2,685 \mathrm{abC}$ \\
EMGOPA-304 & $6,559 \mathrm{bcA}$ & $4,416 \mathrm{abB}$ & $3,129 \mathrm{abcC}$ & $2,678 \mathrm{abC}$ \\
FT-1 & $6,941 \mathrm{abA}$ & $4,878 \mathrm{aB}$ & $3,724 \mathrm{aC}$ & $2,723 \mathrm{aD}$ \\
FT-6 & $7,985 \mathrm{aA}$ & $5,179 \mathrm{aB}$ & $3,526 \mathrm{abC}$ & $2,665 \mathrm{abC}$ \\
\hline Média & 7,084 & 4,714 & 3,362 \\
\hline \multicolumn{5}{c}{ Sensível ao alumínio } \\
EMGOPA-303 & $4,846 \mathrm{dA}$ & $2,797 \mathrm{cB}$ & $2,190 \mathrm{cB}$ & $1,965 \mathrm{abB}$ \\
IAC-13 & $5,530 \mathrm{cdA}$ & $3,252 \mathrm{bcB}$ & $2,060 \mathrm{cC}$ & $1,542 \mathrm{bC}$ \\
Paraná & $7,299 \mathrm{abA}$ & $3,305 \mathrm{bcB}$ & $2,489 \mathrm{bcBC}$ & $2,170 \mathrm{abC}$ \\
SPS-1 & $7,341 \mathrm{abA}$ & $3,369 \mathrm{bcB}$ & $2,629 \mathrm{abcBC}$ & $2,120 \mathrm{abC}$ \\
\hline Média & 6,254 & 3,181 & 2,342 & 1,949 \\
\hline & 1 Valores seguidos de mesma letra, minúscula na coluna e maiúscula na \\
linha, não diferem entre si pelo teste de Tukey, a 5\% de probabilidade.
\end{tabular}

ver diferenças, por grupo de genótipos, quanto à tolerância ao Al.

Os efeitos da ação tóxica do Al na parte aérea das plantas, caracterizados pelo enrolamento ou crestamento de folhas novas e colapso do pecíolo, expressam, na verdade, deficiência de $\mathrm{Ca}$, uma vez que houve decréscimo nos teores desse nutriente e não houve diferença nas concentrações de $\mathrm{Al}$ na parte aérea. Assim, pode-se deduzir, dos resultados obtidos, que o efeito negativo do $\mathrm{Al}$ não ocorre diretamente na absorção de $\mathrm{Ca}$, mas na inibição do crescimento das raízes. A absorção de Ca ocorre predominantemente nos tecidos novos da raiz, antes da suberização (Kirby, 1979). A redução na formação de novas raízes ou no seu crescimento diminui a absorção de $\mathrm{Ca}$, independentemente do efeito direto do Al sobre o processo de absorção. Assim, as menores taxas de crescimento nas raízes, em níveis crescentes de Al, estão associadas a menores taxas de absorção de $\mathrm{Ca}$, sem contudo diferenciar os genótipos tolerantes ao Al.

\section{CONCLUSÕES}

1. A utilização de solução diluída contendo $0,2 \mathrm{mg} \mathrm{L}^{-1}$ de alumínio, combinada com $50 \mathrm{mg} \mathrm{L}^{-1}$ de cálcio, é eficiente na definição das diferenças de tolerância entre os genótipos de soja.

2. A melhor definição das diferenças na tolerância entre os genótipos de soja ao alumínio ocorre no nono dia após o transplante das plântulas.

3. Os genótipos Biloxi, Bragg, BRAS85-1736, BRAS86-3672, BR-13 (Maravilha), BR-37, Cobb, EMGOPA-302, EMGOPA-304 (Campeira), FT-1; FT-5 (Formosa), FT-6 (Veneza), FT-Guaíra, FT-Manacá, IAS 4; IPAGRO-21, Ivaí, MSBR-17 (São Gabriel), OCEPAR 6, Planalto e Tiaraju são fontes de tolerância ao alumínio

4. Menores taxas de crescimento de raízes em genótipos de soja, em níveis crescentes de alumínio, estão associados a menores taxas de absorção de cálcio, sem contudo diferenciar os genótipos tolerantes ao alumínio.

\section{REFERÊNCIAS}

ARMIGER, W.H.; FOY, C.D.; FLEMING, A.L.; CALDWELL, B.E. Differential tolerance of soybean 
varieties to an acid soil high in exchangeable aluminum. Agronomy Journal, Madison, v.60, n.1, p.67-70, 1968.

FONSECA JÚNIOR, N.S. Estudo da herança da tolerância ao alumínio em soja (Glycine $\max (\mathrm{L}$.) Merril), pelo método da hematoxilina. Viçosa : UFV, 1982. 46p. Dissertação de Mestrado.

FONSECA JÚNIOR, N.S.; MARIA, J.; SEDIYAMA, T.; PEREIRA, M.G.; YAMADA, M.M.; TRAGNAGO, J.L. Métodos de detecção visual da sensibilidade ao alumínio em soja. In: SEMINÁRIO NACIONAL DE PESQUISA DE SOJA, 2., 1981, Brasília. Anais. Londrina : Embrapa-CNPSo, 1981. p.678-685.

GREWELING, T. Chemical analysis of plants. Search Agriculture, Ithaca, v.6, p.6-7, 1976.

HAIR JUNIOR, J.F.; ANDERSON, R.E.; TATHAM, R.L. Cluster analysis. In: MULTIVARIATE data analysis with readings. New York : Macmillan, 1987. p.293316.

HANSON, W.D.; KAMPRATH, E.J. Selection for aluminum tolerance in soybeans based on seedling root growth. Agronomy Journal, Madison, v.71, n.4, p.581-586, 1979.

KIRBY, E.A. Maximizing calcium uptake by plants. Communications in Soil Science and Plant Analysis, New York, v.10, n.1/2, p.89-113, 1979.

KONZAK, C.F.; POLLE, E.; KITTRICK, J.A. Screening several crops for aluminum tolerance. In: WORKSHOP ON THE ADAPTATION OF PLANTS TO MINERAL STRESS IN PROBLEM SOILS, 1976, Beltsville. Proceedings. Beltsville : USDA-Plant Stress Laboratory, 1976. p.311-327.

LANCE, J.C.; PEARSON, R.W. Effect of low concentrations of aluminum on growth and water nutrient uptake by cotton roots. Soil Science Society of America. Proceedings, Madison, v.33, n.1, p.9598, 1969.

McALISTER, D.F.; KROBER, O.A. Translocation of food reserves from soybean cotyledons and their influence on the development of the plant. Plant Physiology, Rockville, v.26, n.3, p.525-538, 1951.

MARSCHNER, H.; RICHTER, C.L. Calcium translocation in roots of maize and bean seedlings. Plant and Soil, Dordrecht, v.40, p.193-210, 1974.

MASCARENHAS, H.A.A.; CAMARGO, C.E.O.; FALIVENE, S.M.P. Efeito do alumínio sobre o crescimento de raízes, peso seco da parte aérea e raízes de diferentes cultivares de soja. Bragantia, Campinas, v.43, n.1, p.191-200, 1984a.

MASCARENHAS, H.A.A.; CAMARGO, C.E.O.; FALIVENE, S.M.P. Tolerância de cultivares de soja a dois níveis de alumínio em soluções nutritivas em diferentes concentrações salinas. Bragantia, Campinas, v.43, n.2, p.459-466, 1984b.

MENOSSO, O.G.; PALHANO, J.B.; MARTINS, E.G.; KIIHL, R.A.S.; LANTMAN, A.F. Desenvolvimento de cultivares tolerantes ao complexo de acidez com alta capacidade de extração de fósforo do solo: produção de genótipos tolerantes à acidez do solo. In: EMBRAPA. Centro Nacional de Pesquisa de Soja (Londrina, PR). Resultados de pesquisa de soja 1986/87. Londrina, 1988. p.213-215.

MOORE, D.F.; KRONSTAD, W.E.; METZGER, R.J. Screening wheat for aluminum tolerance. In: WORKSHOP ON THE ADAPTATION OF PLANTS FOR MINERAL STRESS IN PROBLEM SOILS, 1976, Beltsville. Proceedings. Beltsville : USDA-Plant Stress Laboratory, 1976. p.287-295.

MUNNS, D.N. Soil acidity and growth of legume. II. Reactions of aluminum and phosphate in solution and effects of aluminum, phosphate, calcium and $\mathrm{pH}$ on Medicago sativa $\mathrm{L}$. and Trifolium subterraneum L. in solution culture. Australian Journal of Agricultural Research, Collingwood, v.16, n.5, p.743-755, 1965.

NEENAN, $M$. The effects of soil acidity on the growth of cereals with particular reference to the differential reaction of varieties thereto. Plant and Soil, Dordrecht, v.12, p.324-338, 1960.

NORUSIS, M.J. Clustering cases: procedure cluster. In: SPSS: statistical package for the social sciences. New York : McGraw-Hill, 1983. p.265-280.

REID, D.A.; JONES, G.D.; ARMIGER, W.H.; FOY, C.D.; KOCH, E.J.; STARLING, T.M. Differential aluminum response of winter barley varieties and selections in associated greenhouse and field experiments. Agronomy Journal, Madison, v.61, n.2, p.218-222, 1969.

SAPRA, V.T.; MEBRAHTU, T.; MUGWIRA, L.M. Soybean germplasm and cultivar aluminum tolerance in nutrient and Bladen Clay Loam soil. Agronomy Journal, Madison, v.74, n.4, p.687-690, 1982.

SARTAIN, J.B.; KAMPRATH, E.J. Aluminum tolerance of soybean cultivars based on root elongation in solution culture compared with growth in acid soil. Agronomy Journal, Madison, v.70, n.1, p.17-20, 1978. 